ñFunctionalization of surfaces with synthetic oligonucleotidesò Manning, B., Eritja, R. Methods in Molecular Biology, ñNanotechnology in Regenerative Medicine: Methods and Protocols. (J.A. Planell, M. Navarro Eds.) Humana Press, Springer, New York, vol. 811, 89-100 (2012). doi: 10.1007/978-1-61779-388-2_6

\title{
Functionalization of surfaces with synthetic oligonucleotides
}

\section{Brendan Manning and Ramon Eritja*}

Institute for Research in Biomedicine, IQAC-CSIC, CIBER de Bioingeniería, Biomateriales y Nanomedicina (CIBER-BBN), Helix Building, Baldiri Reixac 15, E-

08028 Barcelona, Spain. (phone: +34(93)4039942; fax: +34(93)2045904; e-mail:

$$
\text { recgma@cid.csic.es) }
$$

\begin{abstract}
There is a large interest in the use of nucleic acids covalently bound to surfaces for a variety of biomedical uses; biosensors, microarrays, drug delivery, lab-on-chip devices and gene therapy etc. Most of these applications require the covalent attachment of oligonucleotides via specific reactive groups on both modified oligonucleotide and/or surface. The purpose of this chapter is to provide experimental protocols for the synthesis of oligonucleotides and for the immobilization of these synthetic oligonucleotides onto surfaces such as gold and silicon oxide.
\end{abstract}

Key Words: DNA; nucleic acids; biosensors; surface analysis; oligonucleotides; microarrays

\section{Introduction}


The repair and maintenance of living tissues and organs in vivo is a complex task, regenerative medicine aims to achieve this goal through an amalgamation of synthetic and natural means. The covalent coupling and patterning of biomolecules is expected to play an important role in the development of new biomedical devices (1). Grafting of oligonucleotides to planar surfaces for high throughput parallel screening of biomolecules by dab-on-chipô devices offers the possibility of rapid diagnosis and analysis (2-5). Furthermore short synthetic oligonucleotides can be used to immobilise a variety of organic and inorganic materials onto a surface, such modified surfaces may be used to study the physicochemical properties that affect cellular function (6-9). Also of interest is the development of targeted delivery systems based on nanoparticles or liposomes $(\mathbf{1 0 , 1 1})$. A sophisticated delivery system incorporating bioactive molecules on a surface could provide multiple complementary therapies in a single dose while also sending feedback via reporter molecules (12).

To fabricate devices requiring the attachment of a biomolecule to a substrate, a specific and robust coupling procedure is necessary (13). In this protocol we are focusing on oligonucleotide conjugation to surfaces. Oligonucleotides are short nucleic acid polymers that can easily be synthesised purified and modified within the confines of modern solid phase synthesis. There are two primary substrates identified, gold and silicon oxide, however the coupling methods described are transposable to other suitable surfaces. Covalent coupling to each substrate requires different modifications to the synthetic oligonucleotide. This can be achieved with the use of specialized reagents that are compatible to DNA synthesis conditions and that are able to generate the appropriate functional groups at specific sites on a synthetic oligonucleotide. For a gold surface the modification of choice is the addition of a thiol moiety to the 3ôor 5ôend of the oligonucleotide. The strong affinity of a thiol group for gold results in the 
chemisorption of the oligonucleotide followed by oxidation of the gold to form a covalent bond $(\mathbf{1 4}, \mathbf{1 5})$. When grafting to a silicon oxide substrate there is a greater variety of methods to choose from. Of the current methods it has been shown that use of an amine layer followed by the coupling with a homobifunctional linker, 1,4 phenylenediisothiocyanate, followed by grafting of an amine modified oligonucleotide provides a useful and robust method for the covalent attachment of oligonucleotides (see Note 1) (16-18).

In this regard we describe protocols to synthesise and couple thiol terminated oligonucleotides to gold surfaces and amine terminated oligonucleotides to isothiocyanate modified silicon surfaces.

\section{Fig. 1 near here}

\section{Materials}

\subsection{Synthesis of oligonucleotides carrying amino or disulfide groups at the 5ô or 30̂} ends.

1. Prepare oligonucleotides sequences using solid-phase methodology and 2-cyanoethyl phosphoramidites as monomers. The phosphoramidites of the natural nucleosides are commercially available (see Note 2).

2. Ancillary reagents required for oligonucleotide synthesis are: $0.4 \mathrm{M} 1 H$-tetrazol in ACN (activation); 3\% trichloroacetic acid in DCM (detritylation), acetic anhydride / pyridine /tetrahydrofurane (1: 1: 8) (capping A), $10 \% \mathrm{~N}$-methylimidazole in tetrahydrofurane (capping B), 0.01 M iodine in tetrahydrofurane/ pyridine /water (7: 2: 1) (oxidation). These solutions can be obtained from the same companies that provide phosphoramidites (see Note 2). 
3. Reagent used for the introduction of an amino group at the 3ôend: 3ôamino-modifier C7 CPG (see Fig. 1 and Note 2).

4. Reagent used for the introduction of an amino group at the 5ôend: MMT-amino modifier C6 phosphoramidite (see Fig. 1 and Note 2).

5. Reagent used for the introduction of a disulfide group at the 3ôend: 3 ôthiol-modifier C3 S-S CPG (see Fig. 1 and Note 2).

6. Reagent used for the introduction of a disulfide group at the 5ôend: 5 ôthiol modifier C6 S-S phosphoramidite (see Fig. 1 and Note 2).

7. HPLC solutions are as follows. Solvent A: $5 \%$ acetonitrile in $100 \mathrm{mM}$ triethylammonium acetate $(\mathrm{pH}$ 6.5) and solvent B: $70 \%$ acetonitrile in $100 \mathrm{mM}$ triethylammonium acetate $\mathrm{pH}$ 6.5.

8. Rhodamine derivative carrying a maleimido group: Tetramethylrhodamine 5maleimide (Fluka).

\subsection{Functionalization of gold surfaces with oligonucleotides.}

1. Ultra high purity (UHP) water was purified using a UHQ filtration system and used with resistivity $>18 \Omega \mathrm{cm}^{-1}$.

2. Substrates of gold evaporated on mica (template stripped gold), prepared in house. (see Note 3)

3. 3ôor 5ôDisulfide modified oligonucleotide. (see Note 4)

4. Solution for the immobilization of disulfide-oligonucleotides: 1.0 M Potassium dihydrogen phosphate buffer $\left(\mathrm{KH}_{2} \mathrm{PO}_{4}\right)$.

5. 6-mercaptohexanol solution: $1 \mathrm{mM}$ in UHP water. 
6. Hybridization solution: $\sim 1 \mathrm{nM}$ solution of $10 \mathrm{~nm}$ gold nanoparticles conjugated to complementary oligonucleotide $(\mathbf{1 8}, \mathbf{1 9})$ in $0.01 \mathrm{M}$ phosphate buffer $\mathrm{pH} 7.0,0.3 \mathrm{M}$ $\mathrm{NaCl}$.

7. Washing solution to remove salt from surface: ammonium acetate $0.03 \mathrm{M}$ in UHP water.

\subsection{Functionalization of silicon oxide surfaces with oligonucleotides}

1. UHP water was purified using a UHQ filtration system and used with resistivity $>18$ $\Omega \mathrm{cm}^{-1}$

2. Silicon wafers $\left(\mathrm{Si} / \mathrm{SiO}_{2}\right)$ were purchased from CrysTec $\mathrm{GmbH}$. and were of the type: a) $\langle 100\rangle$ orientation, b) resistance of $1-20 \Omega \mathrm{cm}^{-1}$, c) $200 \mathrm{~nm}$ thick oxide layer on both sides and d) polished on one side.

3. Piranha solution: Concentrated sulfuric acid $\left(\mathrm{H}_{2} \mathrm{SO}_{4}\right)$ and $30 \%$ hydrogen peroxide (30\% $\left.\mathrm{H}_{2} \mathrm{O}_{2}\right)$, (70:30). Caution: Solution dangerously attacks organic matter!

4. RCA (Radio Corporation of America) solution: UHP water; $30 \% \mathrm{H}_{2} \mathrm{O}_{2} ; 33 \% \mathrm{NH}_{4} \mathrm{OH}$ in a ratio of 5:1:1 respectively.

5. APTMS solution: $0.5 \mathrm{mM}$ solution of (3-aminopropyl)-trimethoxysilane (APTMS) in $5 \mathrm{~mL}$ of anhydrous ethanol.

6. PDITC solution: $0.2 \%$ solution of 1,4-diphenylenediisothiocyanate (PDITC) in $10 \%$ pyridine/DMF.

7. 3ô or 5ôamino modified oligonucleotide.

8. Solution for the immobilization of amino-oligonucleotides: $10 \mu \mathrm{M}$ oligonucleotide$\mathrm{NH}_{2}$ in $1 \mathrm{M}$ Tris- $\mathrm{HCl}(\mathrm{pH} 7.0)$ with $1 \% \mathrm{~N}, N$-diisopropylethylamine.

9. Amine passivation solution: $50 \mathrm{mM}$ 6-amino-1-hexanol, $150 \mathrm{mM} \quad \mathrm{N}, N$ diisopropylethylamine in UHP water. 
10. 20x SSC concentrate (Saline Sodium Citrate: $0.3 \mathrm{M}$ sodium citrate, $3.0 \mathrm{M} \mathrm{NaCl}, \mathrm{pH}$ 7.0)

11. Hybridization solution: $20 \mu \mathrm{M}$ solution of the rhodamine labelled oligonucleotide in $5 \times$ SSC containing $1 \%$ SDS and $20 \%$ formamide.

\subsection{Functionalization of glass slides with oligonucleotides.}

1. Glass slides functionalized with poly-(Lys) (MENZEL-GLASER, P4981-602677$\mathrm{CE})$.

2. PDITC solution: $0.2 \%(\mathrm{w} / \mathrm{v})$ solution of $1,4-$ phenylene diisothiocyanate (PDITC) in $10 \%$ pyridine / dimethylformamide.

3. Solution used for the immobilization of amino-oligonucleotides: $10 \mu \mathrm{M}$ oligonucleotide in 0.1 M Borate-Na buffer, $\mathrm{pH}$ 8.0.

4. Passivation solution: $1 \mathrm{~g}$ of succinic anhydride in $50 \mathrm{~mL}$ of $N$-methylpyrrolidinone and 5.6 mL 0.2 M Borate-Na buffer, $\mathrm{pH}$ 8.0.

5. Prehybridization solution: a solution of $1 \%$ bovine serum albumin (BSA), in 6x SSC (saline sodium citrate, see 2.3.10).

6. Hybridization solution: $20 \mu \mathrm{M}$ solution of the rhodamine labeled oligonucleotide in 5 x SSC containing $1 \%$ SDS and $20 \%$ formamide.

\section{Methods}

As discussed, there is a large interest in the use of biomolecules covalently bound to surfaces for a variety of biomedical applications. Covalent attachment of oligonucleotides to surfaces requires modification of the oligonucleotides and often modification of the surface utilised. There are a large number of protocols to immobilize oligonucleotides to surfaces $(3-5,13)$. In this work we describe robust methods for the 
immobilization of synthetic oligonucleotides onto gold, glass and silicon oxide surfaces. As recent examples of the potential applications developed from our group using the methodologies described here we underline the formation of patterns on silicon oxide substrates using hairpin oligonucleotides carrying photolabile groups (18) and the use of thiolated oligonucleotides to form 2D-DNA arrays on gold surfaces (15).

\section{Fig. 2 near here}

\subsection{Synthesis of modified oligonucleotides}

1. Prepare oligonucleotide sequences were prepared using solid-phase methodology and 2-cyanoethyl phosphoramidites as monomers. Synthesize were performed on a DNA synthesizer using either 0.2 or $1 \mu$ mol scales.

2. The solid supports used more frequently for the introduction of amino or thiol groups at the 3ôends are shown in Fig. 1. For the introduction of an amino group use the 30̂ amino-modifier C7 CPG. This support is a controled pore glass (CPG) support functionalized with an amino-diol, having the amino group protected with the 9fluorenylmethyloxycarbonyl (Fmoc) group (see Note 5) (20). For the introduction of the thiol or disulfide group use the 3ôthiol-modifier C3 S-S CPG. This support is a CPG support functionalized with the hydroxypropyldisulfide protected with the dimethoxytrityl (DMT) group.

3. The introduction of amino or disulfide groups at the 5ôend needs the use of a special phosphoramidite (Fig. 1). For the introduction of an amino group at the 5ôend use the MMT-amino modifier C6 phosphoramidite (21). This reagent is a derivative of 6aminohexanol with the monomethoxy (MMT) group for the protection of the amino 
group (see Note 6). For the introduction of a thiol or disulfide group at the 5ôend use the 5ôthiol modifier C6 S-S phosphoramidite.

4. After the assembly of sequences, deprotect in $30 \%$ aqueous ammonia solution overnight at $55^{\circ} \mathrm{C}$. If oligonucleotides carrying a free thiol group are required deprotect with $1 \mathrm{~mL}$ of $0.1 \mathrm{M}$ dithiothreitol (DTT) in $30 \%$ aqueous ammonia solution (see Note 4).

Purify oligonucleotides carrying amino groups by reverse-phase HPLC. HPLC solutions are described above (Subheading 2.1). Columns: Nucleosil 120C18 (10 $\mu \mathrm{m}), 200$ x 10 mm. Flow rate: $3 \mathrm{~mL} / \mathrm{min}$. Conditions A: 20 minutes linear gradient from $15-80 \%$ (DMT on). Conditions B: 20 minutes linear gradient from 0-50\% B (DMT off). The DMT or MMT-oligonucleotides are purified using conditions A (22). The major peak containing the DMT- or MMT-oligonucleotide will elute around 10-12 minutes.

6. Collect the eluates and concentrate to dryness and treat the residue with $80 \%$ acetic acid in water. After $30 \mathrm{~min}$, add $1 \mathrm{~mL}$ of water and extract the acetic acid with ethyl ether (3 times). Repurify the resulting aqueous solution by HPLC using conditions B.

7. Purify oligonucleotides carrying free thiol groups and DTT in a NAP-5 or NAP-10 (Sephadex G25) column elute with water according to the manufacturerô protocol. Deptrotected oligonucleotides with a free thiol should be used immediately without further purification.

8. The synthesis of rhodamine-labelled complementary oligonucleotide is performed by reaction of a free thiol-oligonucleotide with tetramethylrhodamine 5-maleimide (see Note 7). Add a large excess of maleimido-rhodamine (1mg) to the NAP purified deprotected thiol-oligonucleotide $(0.5 \mathrm{~mL})$ and allow shake gently for 4 hours at room temperature. Concentrate the solution to dryness and then redissolve in $0.5 \mathrm{~mL}$ water. Purify in a NAP-5 (Sephadex G-25) column eluting with water to separate the desired 
conjugate from the excess maleimido rhodamine. Purify the conjugate further by HPLC using conditions A (DMT on). The product carrying the fluorescent oligonucleotide (retention time $8 \mathrm{~min}$ ) is isolated in $\sim 70 \%$ yield.

\section{Fig. 3 near here}

\subsection{Immobilization of oligonucleotides on gold substrates}

1. Depending on gold substrate chosen cleave gold substrate from support or clean according to suppliers guidelines (see Note 3).

2. Drop cast $10 \mu \mathrm{L}$ a $3 \mu \mathrm{M}$ solution of the disulfide oligonucleotide in immobilisation buffer onto the gold surface. Ensuring the drop covers the required area.

3. Incubate the substrate in a humid environment for 10 hours. Following incubation wash with UHP water to remove any non-covalently bound oligonucleotides.

4. Drop cast $15 \mu \mathrm{L}$ of $1 \mathrm{mM}$ 6-mercaptohexanol onto the gold surface. Incubate in a humid environment for 1 hour. Wash the solution with UHP water. Mercaptohexanol passivates the gold surfaces and improves hybridisation efficiency.

5. The conjugated oligonucleotide can then be hybridised with a nanoparticle label. Drop cast a solution of $10 \mathrm{~nm}$ gold particles carrying a complementary oligonucleotide on the gold surface for 2 hours in a humid environment at room temperature. Wash three times $0.3 \mathrm{M} \mathrm{NaCl}, 0.01 \mathrm{M}$ phosphate buffer, $\mathrm{pH} 7$ and once with $0.03 \mathrm{M}$ ammonium acetate.

\section{Fig. 4 near here}

\subsection{Functionalization of self-assembled monolayers (SAM) on silicon oxide surfaces}


1. Cut silicon wafers into squares of approximately $1 \mathrm{~cm} \times 1 \mathrm{~cm}$ using a diamond tipped scriber. Rinse the substrates with ethanol to remove of any dust that was produced during the cutting process.

2. Immerse the cut silicon in piranha solution at $90-100{ }^{\circ} \mathrm{C}$ for 60 minutes. Once cooled, rinse off the piranha solution with UHP water.

3. Agitate in an ultrasonic bath in RCA solution for 60 minutes. Sonication in RCA at this stage functionalises the surface with hydroxyl groups to allow monolayer formation. Rinse the substrates in UHP water to finish the cleaning procedure. The substrates are then stored in UHP water and used within 2 days to minimise loss of surface hydroxyl groups.

4. Transfer the cleaned silica substrates from UHP water to anhydrous ethanol by stepwise exchange $\left(\mathrm{H}_{2} \mathrm{O}: \mathrm{EtOH}\right)-3: 1,2: 2,1: 3,0: 4$ and finally anhydrous ethanol. In a nitrogen atmosphere immerse the substrates in APTMS solution and sonicate at room temperature for 1 hour. Rinse the substrates for 20 seconds each with ethanol and chloroform. Sonicate the wafers twice in fresh ethanol for 5 minutes followed by a final sequential rinsing with ethanol and chloroform. Dry samples under a steady stream of nitrogen and cure at $120{ }^{\circ} \mathrm{C}$ for 30 minutes under vacuum to promote cross-linking of the SAMôs.

5. Incubate APTMS functionalised silicon wafers for 2 hours in PDITC solution. After incubation, wash with methanol and acetone and stored in vacuum desiccators.

6. Deposit a drop of oligonucleotide- $\mathrm{NH}_{2}$ solution onto substrate and incubate at $37{ }^{\circ} \mathrm{C}$ for 2 hours. Wash samples with 1\% ammonium hydroxide and copious amounts of UHP water. Immerse wafers in amine passivation solution for 2 hours. Dry under a steady stream of $\mathrm{N}_{2}$ and store under vacuum in a desiccator. 
7. Drop cast fluorescently labelled complementary in hybridisation buffer on the wafer. Incubate for 2 hours at room temperature. Wash with 5x SSC and finally with 1x SSC.

\section{Fig. 5 near here}

\subsection{Immobilization of oligonucleotides on glass substrates}

1. Clean amine functionalised glass slides 10 times with acetone and dry for 45 minutes at $90^{\circ} \mathrm{C}$.

2. Treat glass slides with PDITC solution for 2 hours at room temperature. Wash glass slides with methanol (3x) and acetone (2x). Use glass slides immediately.

3. Add the desired amount of amino-oligonucleotide immobilization solution to the glass slide. Incubate for 2 hours at $37^{\circ} \mathrm{C}$ and then wash with $1 \% \mathrm{NH}_{4} \mathrm{OH}$ and water $(30$ $\mathrm{mL} \times 5)$.

4. Agitate in succinic anhydride passivating solution for 2 hours. This post-processing is required to passivate the charged surface. Rinse by agitating in UHP water and ethanol $96 \%$, centrifuge for 6 min at 1500 r.p.m. and dry with a steady stream of argon.

5. Prehybridize with the bovine serum albumin solution for 10 minutes at room temperature. Drop cast the hybridization solution to the requires area and incubate in a humid environment for 2 hours at room temperature. Measure the fluorescence profile on a fluorescence scanner.

\section{Notes}

1. Outside the scope of this short procedure is the electrostatic binding of DNA to surfaces and also the use of different types of substrates. The type of surface chosen is dependent on the required use and the type of characterisation envisaged. Described 
here is the fabrication of very flat amine monolayers on high grade silicon wafers; this method facilitates characterisation by AFM. Using prefabricated amine terminated glass slides may be sufficient if one is only using fluorescent microscopy as detection method.

2. There are several companies that provide regular phosphoramidite, ancillary reagents and solid supports for oligonucleotide synthesis such as Applied Biosystems and Sigma. In addition to standard reagents, more elaborate phosphoramidites and solid supports can be found at companies specializing in oligonucleotide reagents such as Link technologies (Scotland), Glen Research (Sterling, VI, USA), Berry \& Associates (Dexter, MI, USA), TriLink Biotechnologies (San Diego, CA, USA), Nedken (Foster City, CA, USA), ChemGenes (Wilmington, MA, USA), Prime Synthesis (Aston, PA, USA) and Metkinen (Finland).

3. There are many suitable gold substrates commercially available. Suitability of one substrate over another depends on application. Some suppliers include Arandee (Wether, Germany), Asylum Research (Santa Barbara, CA, USA) and Sigma Aldrich. Some require cleaning steps to remove organic and inorganic build-up, such cleaning procedures are usually supplied by the manufacturer. During our research we have used gold coated mica substrates prepared in house using a method first described by Hegner et al. (23), which have the advantage of not requiring a cleaning step and can be used ás isôwhen cleaved from a support.

4. It is worth discussing the use of disufide and thiol modified oligonucleotides. Disulfide oligonucleotides such as those in Fig. $\mathbf{2}$ have the advantage that they are stable to long-term storage and spontaneously react with gold surfaces. Alkylthiolprotected thiol strands normally bear disulfide protecting groups that must be removed before conjugation. This is done by adding a cleavage agent such as DTT or DTE which 
cleaves the disulfide bond; however DTT or DTE needs to be removed from the free thiol for it to react with a gold surface or any other suitable reactive group. This is done by size exclusion gravity chromatography (NAP-5 and NAP-10 columns). Often if a thiol oligonucleotide is purchased from a commercial supplier it will contain DTT or DTE.

5. During the addition of the MMT-amino-modifier C6 it is important to avoid the capping reaction because the MMT-amino group can be acetylated giving an unreactive acetyl derivative of the oligonucleotide $(\mathbf{2 4}, \mathbf{2 5})$. Moreover, some authors described low efficiency of the removal of the MMT group. To solve this problem new amino linkers have been described (26) but they are not commercially available. Alternatively the trifluoroacetyl (TFA) derivative of 6.aminohexanol (50̂TFA-amino modifier C6 phosphoramidite) can be used. The TFA group is removed during the ammonia so HPLC purification based on the presence of the MMT group is not possible.

6. Some authors have described that the Fmoc group protecting the amino function is partially lost during the synthetic process and becomes acetylated during capping conditions (27). In shorter sequences we have also found a side product (5-10\%) with a molecular weight 100 units higher than expected. We assign this side product to a succinylated amino oligonucleotide. This side compound can be produced by internal aminolysis of the succinyl ester. The use of a commercially available phthaloyl linker (3ôPT-amino modifierC6 CPG) is a good alternative to increase the yield of final product (27).

7. In addition to the conjugation with fluorescent derivatives carrying maleimido groups there are several phosphoramidites and solid supports that can be found at companies specializing in oligonucleotide reagents that allow the rapid preparation of fluorescently-labelled oligonucleotides. 


\section{Acknowledgments.}

This work is supported by Spanish Ministry of Education (NAN2004-09415-C05-03, BFU2007-63287/BMC), Generalitat de Catalunya (2005/SGR/00693), Instituto de Salud Carlos III (CIBER-BNN), and European Communities (NANO-3D NMP4CT2005-014006, DYNAMO contract 028669 (NEST), FUNMOL NMP4-SL-2009213382). B.M. thanks the SFI-CRANN/UCD for the predoctoral fellowship. We are thankful to Elena Martínez and Xavier Sisquella of Scientific Park of Barcelona (PCB) for providing us template stripped gold.

\section{References}

1. Engel, E., Michiardi, A, Navarro, M., Lacroix, D., and Planell J.A. (2007) Nanotechnology in regenerative medicine: the materials side. Trends Biotech 26, 39-47

2. Cheng M.M-C., Cuda, G. Bunimovich, Y.L., Gaspari, M., Heath, J.R., Hill H.D., Mirkin, C.A., Nijdam, A.J., Terracciano, R., Thunday, T., and Ferrari M. (2006) Nanotechnologies for biomolecular detection and medical diagnostics. Curr Op Chem Biol 10, 11-9.

3. Sassolas, A., Leca-Bouvier, D., and Blum, L. J. (2008) DNA biosensors and microarrays. Chem Rev 108, 109-139.

4. Liu, J., Cao, Z., and Lu, Y. (2009) Functional nucleic acids sensors. Chem Rev 109, 1948-1998.

5. Dittrich P.S. and Manz, A. (2006) Lab-on-a-chip microfluidics in drug discovery. Nat Rev Drug Discov 5, 210-8

6. Ito, Y. (2000) Surface micropatterning to regulate cell functions. Biomaterials 20, 2333-2342. 
7. Stevens, M.M., and George, J.H. (2005) Exploring and engineering the cell surface interface. Science 310, 1135-8.

8. Wilkins, C.D.W., Riehle, M., Wood, M., Gallagher, J., and Curtis, A.S.G. (2002) The use of nanomaterials patterned on a nano- and micro-metric scale in cellular engineering. Mater Sci Eng C 19, 263-9.

9. Teixeira, A.I., McKie, G.A., Foley, J.D., Bertics, P.J., Nealey, P.F., and Murphy, C.J. (2006) The effect of environmental factors on the response of human corneal epithelial cells to nanoscale substrate topography. Biomaterials 27, 3945-3954.

10. Chertok, B., Moffat, B.A., David, AE, Yu, F., Bergemann, C, Ross, B.D. and Yang, V.C. (2008) Iron oxide nanoparticles as a drug delivery vehicle for MRI monitored magnetic targeting of brain tumours. Biomaterials 29, 487-496.

11. Murthy, N., Campbell, J., Fausto, N., Hoffman, A.S. and Stayton, P.S. (2003) Design and synthesis of $\mathrm{pH}$-responsive polymeric carriers that target uptake and enhance the intracellular delivery of oligonucleotides. J Control Release 89, 365-374.

12. Chertok, B., Moffat, B.A., David, A.E., Yu, F., Bergemann, C., Ross, B.D., and Yang, V.C. (2008) Iron oxide nanoparticles as a drug delivery vehicle for MRI monitored magnetic targeting of brain tumors. Biomaterials 29, 487-496.

13. Pirrung, M.C. (2002) How to make a DNA chip. Angew Chem Int Ed 41, 12761289.

14. Huang, E., Satjapipat, M., Han, S., and Zhou, F. (2001) Surface structure and coverage of an oligonucleotide probe tethered onto a gold substrate and its hybridization efficiency for a polynucleotide target. Langmuir 17, 1215-1224.

15. Garibotti, A.V., Sisquella, X., Martínez, E., and Eritja R (2009) Assembly of twodimensional DNA crystals carrying $\mathrm{N}^{4}$-[2-(tert-butyldisulfanyl)ethyl]-cytosine residues. Helv Chim Acta 92, 1466-1472. 
16. Manning, M., and Redmond, G. (2005) Formation and characterization of DNA microarrays at silicon nitride substrates. Langmuir 21, 395-402.

17. Manning, M., Galvin, P., and Redmond, G. (2002) A robust procedure for DNA microarray fabrication and screening in the molecular biology laboratory. Am Biotech Lab 20, 16-7.

18. Manning, B., Leigh, S.J., Ramos, R., Preece, J., and Eritja, R. (2010) Fabrication of patterned surfaces by photolithographic exposure of DNA-hairpins carrying a novel photolabile group. J Exp Nanoscience 5, 26-39.

19. Mirkin, C.A., Letsinger, R.L., Mucic, R.C., and Storhoff, J.J. (1996) A DNA-based method for rationally assembling nanoparticles into macroscopic materials. Nature $\mathbf{3 8 2}$, 607-609.

20. Nelson, P.S., Kent, M., and Muthini, S. (1992) Oligonucleotide labelling methods. 3. Direct labeling of oligonucleotides employing a novel, non-nucleosidic, 2aminobutyl-1, 3-propanediol backbone. Nucleic Acids Res 20, 6253-9.

21. Sinha, N.D., and Cook, R.M. (1988) The preparation and application of functionalised synthetic oligonucleotides. III Use of H-phosphonate derivatives of protected aminohexanol and mercaptopropanol or mercaptohexanol. Nucleic Acids Res 16, 2659-2669.

22. Güimil García, R., and Eritja, R. (1997) Preparation, purification and analysis of synthetic oligonucleotides suitable for microinjection experiments. Microinjection and transgenesis. Strategies and protocols (A. Cid-Arregui, A. García-Carrancá, Eds) Springer Laboratory Manuals, Springer, Berlin pp 95-112.

23. Hegner, M., Wagner, P., and Semenza, G. (1993) Ultralarge atomically flat template-stripped Au surfaces for scanning probe microscopy. Surf Sci 291, 39-46. 
24. Zaramella, S., Yeheskiely, E., and Strömberg, R. (2004) A method for solid-phase synthesis of oligonucleotide 5ôpeptide-conjugates using acid-labile alpha-amino protection. J Am Chem Soc 126, 14029-14035.

25. Ocampo, S. M., Albericio, F., Fernández, I., Vilaseca, M., and Eritja, R. (2005) A straightforward synthesis of 5ôpeptide oligonucleotide conjugates using $\mathrm{N}^{\alpha}$-Fmocprotected amino acids. Org Lett 7, 4349-4352.

26. Kojima, N., Takebayashi, T., Mikami, A., Ohtsuka, E., and Komatsu, Y. (2009) Efficient synthesis of oligonucleotide conjugates on solid-support using an (aminoethoxycarbonyl)aminohexyl group for 5ôterminal modification. Bioorg Med Chem Lett 19, 2144-7.

27. Petrie, C. R., Reed, M. W., Adams, A. D., and Meyer, R. B. Jr (1992) An improved CPG support for the synthesis of 3ôamine tailed oligonucleotides. Bioconjug Chem $\mathbf{3}$, $85-7$. 
Fig. 1. Phosphoramidites and solid supports used in the preparation of oligonucleotides carrying amino or thiol groups at their $3 \hat{o}$ or $5 \hat{\theta} e n d s$.
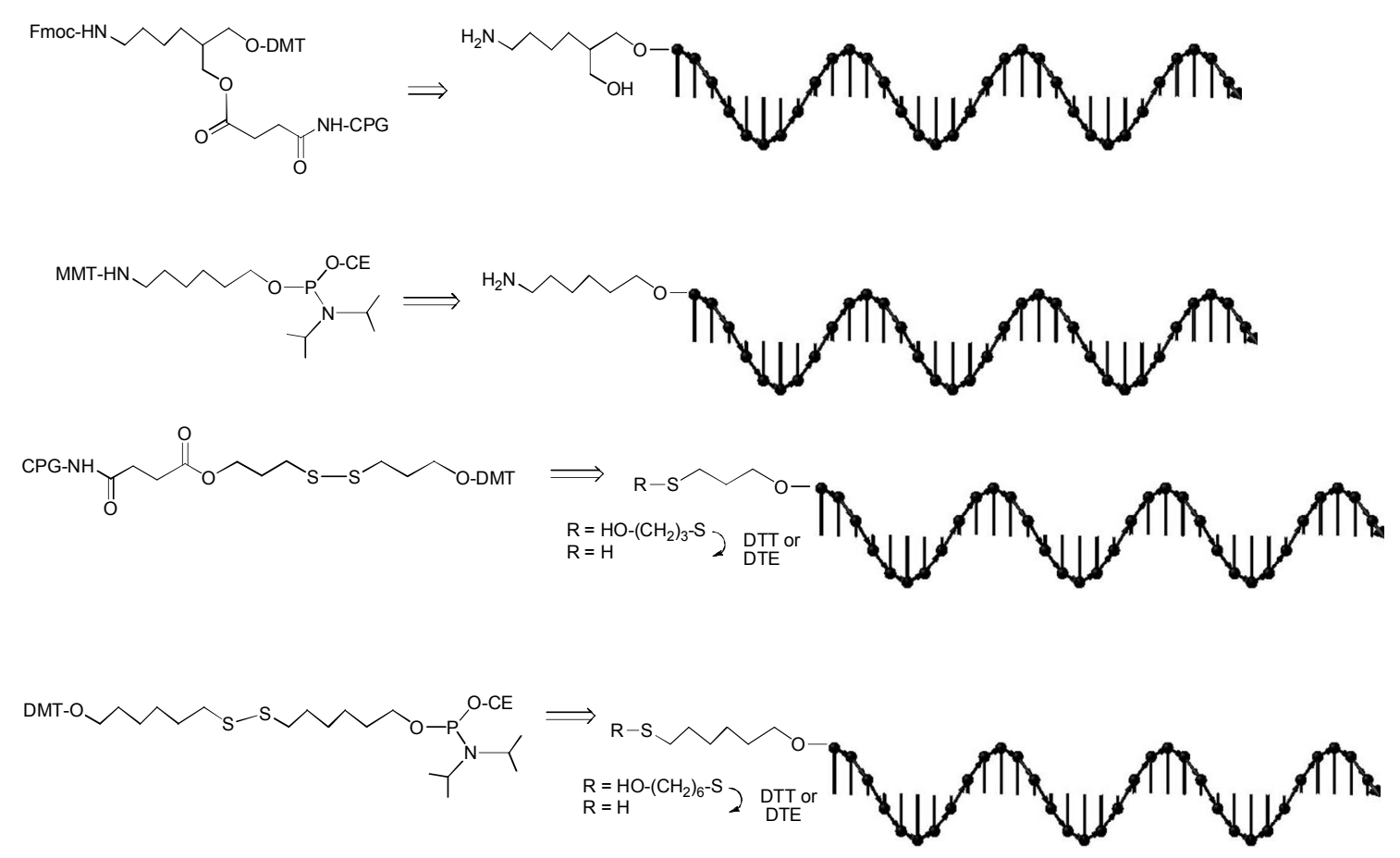
Fig. 2. Reaction of a thiol-oligonucleotide with tetramethylrhodamine 5-maleimide to yield a rhodamine labelled oligonucleotide.

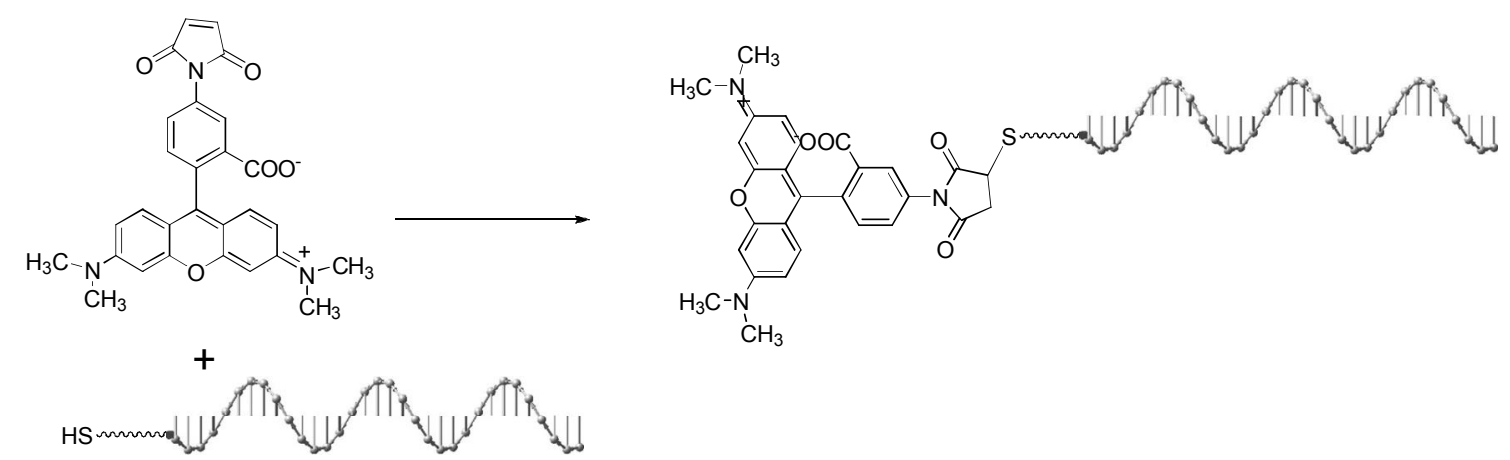


Fig. 3. Scheme of the grafting of an oligonucleotide to a silicon wafer. Wafers are incubated with a solution of APTMS (step 1) followed by crosslinking (step 2) to form an amine terminated monolayer. Reaction with PDITC (step 3) yields a thiocyante terminated layer which in turn can be reacted with an amine functionalised oligonucleotde (step 4). Hybridisation with a labelled complementary oligonucleotide demonstrates the successful grafting of the amine oligonucleotide to the surface.

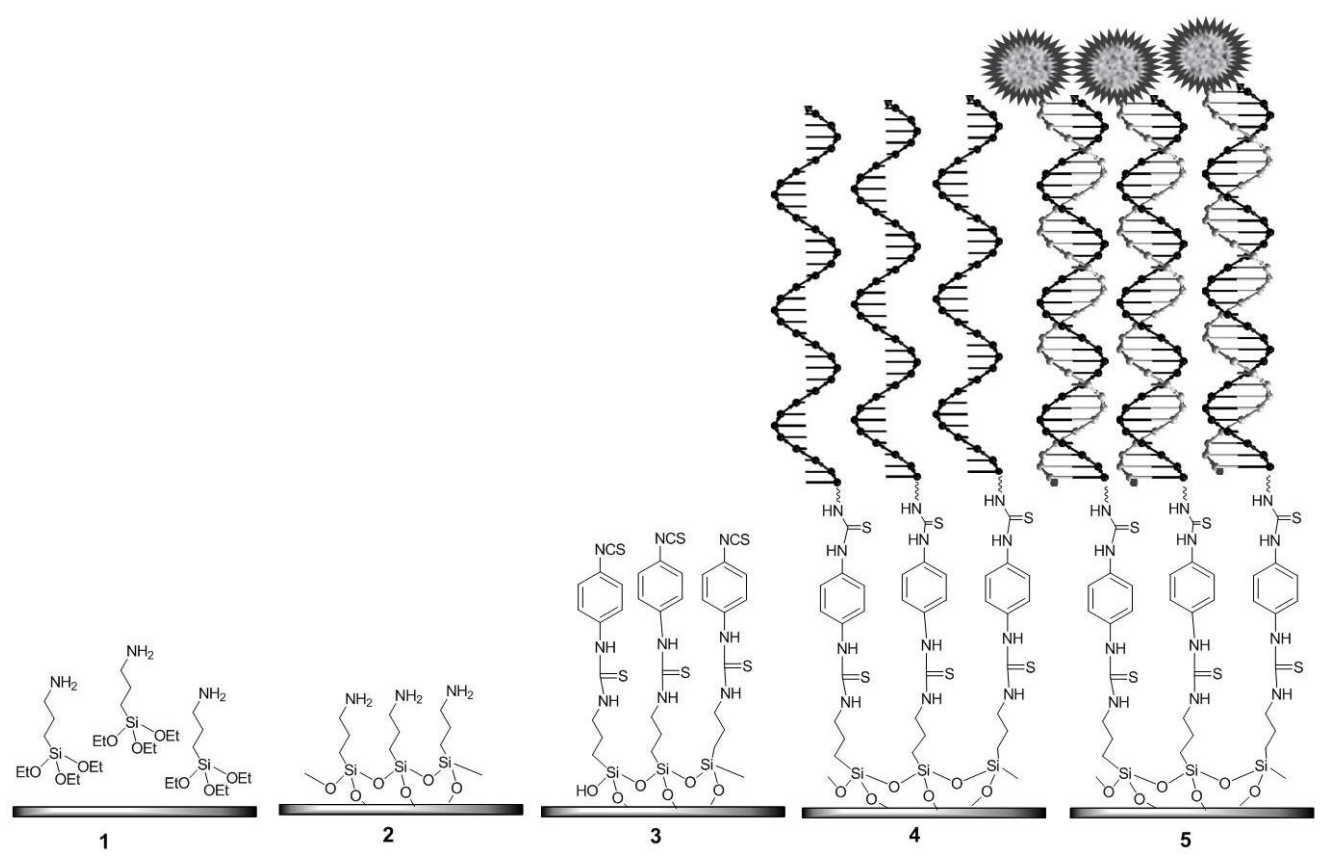


Fig. 4. AFM image of a gold substrate functionalized with an oligonucleotide and hybridized with complementary oligonucleotide labelled with $10 \mathrm{~nm}$ gold nanoparticles.

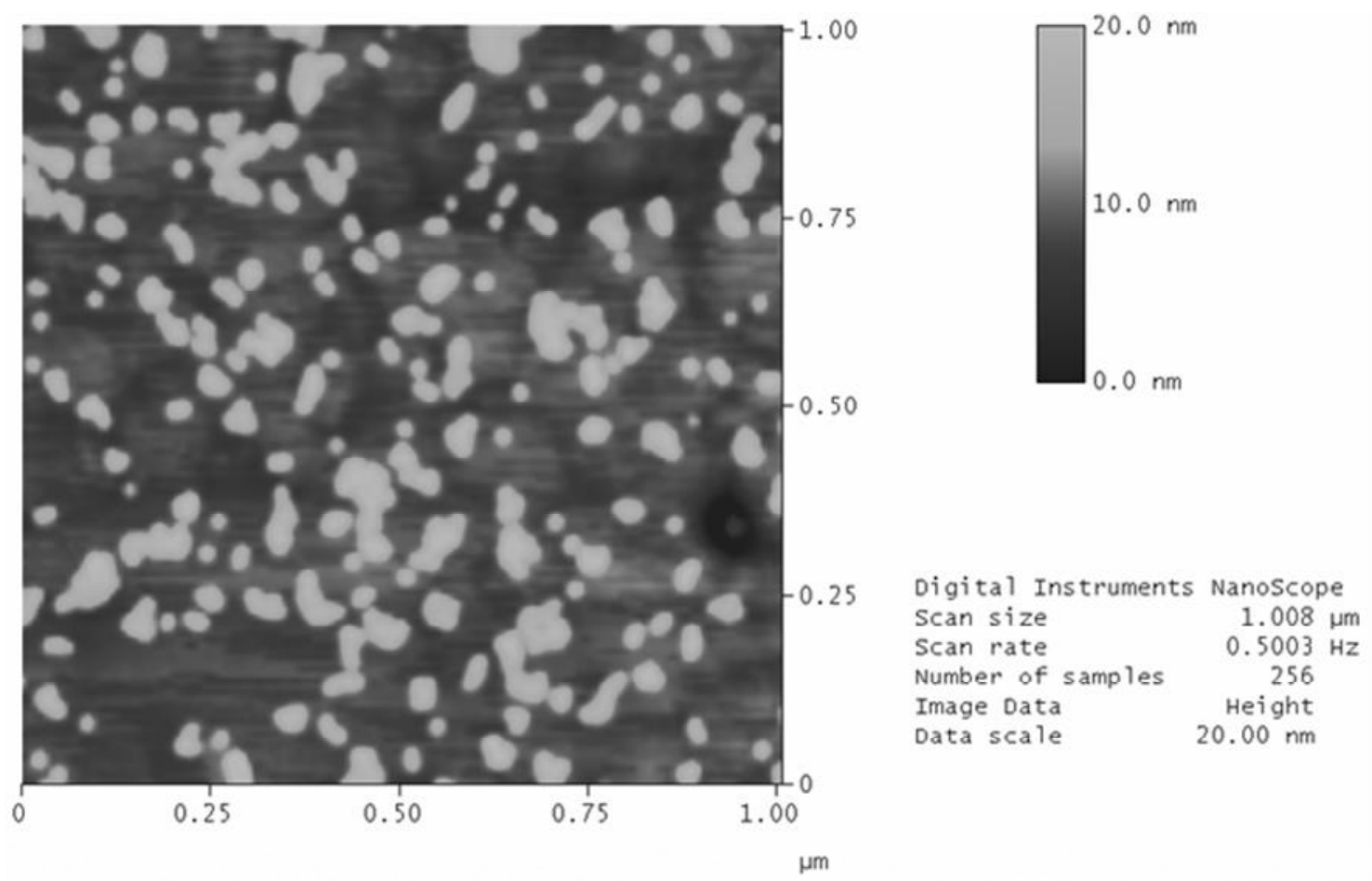


Fig. 5. Fluorescent image of a silicon oxide substrate functionalized with an oligonucleotide (right) and hybridized with complementary oligonucleotide labelled with Rhodamine (left). Inset in the right side shows the AFM image of the silicon surface functionalized with double stranded oligonucleotides (after hybridization).

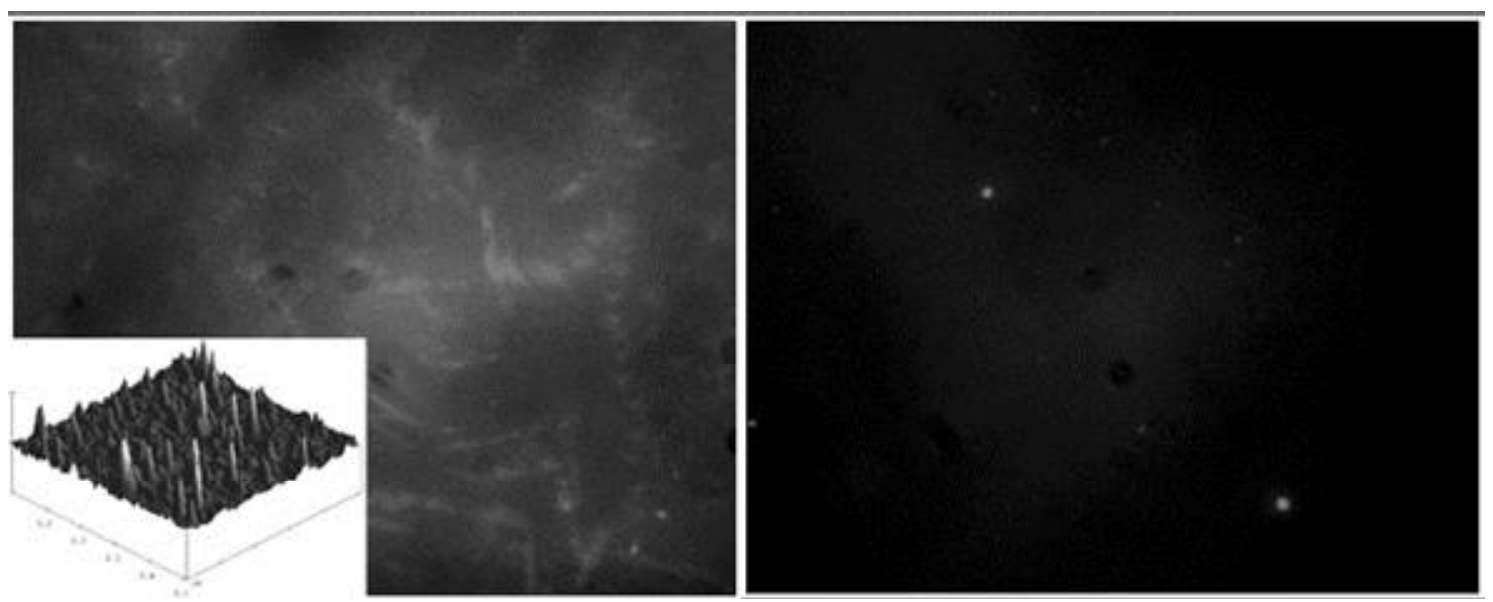

